\title{
Massive Thrombophlebitis as a Complication after Carpal Tunnel Release: Comorbid Factors?
}

Liselore Maeckelbergh ${ }^{1 *}$,Jan Noyez ${ }^{1}$, and Arne Decramer ${ }^{1}$

${ }^{1}$ Department of Orthopaedic Surgery, University of Leuven, Pellenberg, Belgium.

*Corresponding Author : Liselore Maeckelbergh, Department of Orthopaedic Surgery, University of Leuven, Pellenberg, Belgium.

E-mail: liselore.maeckelbergh@hotmail.com

Received date: November 02, 2018;Accepted date : December 10,2018; Published date: December $12,2018$.

Citation: Liselore Maeckelbergh, Jan Noyez,Arne Decramer. Massive Thrombophlebitis as a Complication after Carpal Tunnel Release: Comorbid Factors?. J Orthopaedics and Surgical Sports Medicine. Doi: 10.31579/2641-0427/011.

Copyright : @ 2018 Liselore Maeckelbergh. This is an open-access article distributed under the terms of The Creative Commons Attribution License, which permits unrestricted use, distribution, and reproduction in any medium, provided the original author and source are credited.

\section{Case Report}

A 68-year old male cyclist was seen at the consultation seven days after upper limb surgery.

Seven days before the patient had an endoscopic carpal tunnel release under locoregional anesthesia. We performed a single-portal technique as described by Agee. The patient was positioned in dorsal decubitus with this right arm on a side table. The arm tourniquet was inflated to a pressure $100 \mathrm{mmHg}$ above systolic blood pressure. The tourniquet was inflated after draping and was released after suture. The vertical incision was located radiocarpal along the ulnar border of the musculus palmaris longus. A proximal based flap of the superficial fascia is created and elevated. A blunt probe was used to gently probe the undersurface of the ligament. Sequentially larger dilators are passed into the carpal tunnel. The arthroscope with the blade is passed in and the dissection of the ligamentum transversum carpi is made. The incision is closed with resorbable sutures after which a compressive bandage is applied.

The patient presented with pain at the ulnar side of the forearm distal of the cubital tunnel seven days after surgery. The patient had been using Testocap $^{\circledR}$ (testosterone undecanoate), otherwise no specific medical history. Physical examination showed only a painful nodule at the forearm. There were no signs of infections or hematoma. Technical investigation with ultrasound shows a large superficial phlebothrombosis of the vena basilica with a length of 5 centimeters proximal of the elbow, reaching over the elbow joint 15 centimeters distally with limited inflammation of the subcutaneous fat. After consultation with the vascular surgeons the treatment consisted of low molecular weight heparins (Fraxodi ${ }^{\circledR} 0.6 \mathrm{ml}$ ) for 10 days, after which Fraxiparine ${ }^{\circledR} 0.6 \mathrm{ml}$ was given.

Three weeks later the patient was seen again. Ultrasound showed limited thickening of the vena basilica, reaching over the elbow joint. No signs of inflammation. We continued treatment with low molecular weight heparins for another three weeks.

Keywords: massive thrombophlebitis; carpal tunnel release; upper limb; under locoregional anesthesia

\section{Literature}

Upper-extremity deep venous thrombosis (DVT), although not as common as its lower-extremity counterpart, is a clinical entity with potentially devastating complications. A small percentage of all DVT cases involve the upper extremity, and some of these cases are complicated by pulmonary embolism. Prompt diagnosis with duplex ultrasonography and subsequent anticoagulation are the gold standards for identification and treatment. The majority of these cases are secondary to medical comorbidities. Although rare, several case reports of orthopedic-related upper-extremity DVT are presented in the literature.

Carpal tunnel syndrome (CTS) is the most frequently encountered compression neuropathy of the upper extremity with a prevalence of 1 percent in the general population [1].

CTS is caused by compression of the median nerve in the carpal tunnel at the wrist and produces pain, paresthesia and hypoesthesia in the hand. The severity of CTS ranges from mild to severe.

Mild CTS presents with intermittent symptoms of paresthesia and numbness, often at night. Severe CTS may cause permanent atrophy of the thenar muscles innervated by the median nerve and permanent loss of sensation in the median nerve distribution in the hand. CTS is a clinical diagnosis. Electrophysiological tests (nerve conduction studies) are performed to support the clinical diagnosis.

The first open carpal tunnel release is credited to Learmonth in 1929 and was later popularized by Phalen et al. in the 1950s.

Auctores Publishing - Volume1-007 www.auctoresonline.org
The open carpal tunnel is seen as the standard of care for the treatment of carpal tunnel syndrome because of extensive anatomical exposure. This results in an efficacious and safe release of the transverse carpal ligament. Commonly reported complications of the open technique include hypertrophic or painful scar, pillar pain, slow return of pinch and grip strength and slow return to activities of daily living or work. Endoscopic carpal tunnel release had been developed in principle to obviate these problems. The initial description was in 1989 by Okutsu et al. Endoscopic approaches appear to offer potential advantages related to reduced scarring, minimized postoperative pain, less initial loss of grip and pinch strength, faster recovery and better cosmesis [2,3]. There is discussion about the safety regarding this technique with reports of an increased rate of major neurovascular injuries.

Superficial thrombophlebitis can occur spontaneously, especially in the lower extremities in the greater saphenous vein, or as a complication of medical or surgical interventions. Although the ethology is frequently obscure, superficial venous thrombosis is most often associated with one of the components of the Virchow triad: intima damage, stasis or turbulent flow, or changes in blood constituents. The superficial thrombophlebitis presents as redness and tenderness along the course of the vein, usually accompanied by swelling. Bleeding also can occur at the site of a varicose vein. Superficial thrombophlebitis of the upper extremities usually occurs at infusion or trauma sites. A deep venous thrombosis should be excluded. Treatment for superficial thrombophlebitis is aimed at patient comfort and preventing involvement of the deep veins. 
Deep vein thrombosis (DVT) can cause severe morbidity and mortality [3-5]. DVT and consequent pulmonary embolism (PE) usually occur after pelvic fractures, prolonged lower extremity surgeries and longterm immobilization periods. It is seen less common $(1-2 \%$ of all DVTs) in the upper than the lower extremities.

Upper extremity deep venous thrombosis refers to thrombosis of the brachial, subclavian and/or axillary veins and accounts for up to $11 \%$ of all cases of deep venous thrombosis. Usually, the thrombotic process involves more than one venous segment, with the subclavian vein most frequently affected. The internal jugular vein, the brachio- cephalic vein and the basilic vein each are additionally involved in $20-30 \%$ of patients with upper extremity deep venous thrombosis.

Upper extremity deep venous thrombosis can be classified as primary of secondary. It is classified as secondary in the presence of known endogenous or exogenous risk factors. The secondary form accounts for about two-thirds of the upper extremity deep venous thrombosis and usually affects older patients with severe comorbidities. The most important risk factor for upper extremity deep venous thrombosis is the presence of an indwelling central venous catheter, malignancy, upper extremity immobilization by plaster casts or following arm surgery.

Patients suffering from upper extremity deep venous thrombosis most commonly present with edematous swelling of the affected arm and/or upper extremity discomfort or pain. Other clinical features such as cyanosis, visible collateral veins at the shoulder girdle or jugular distension are less frequently found.

Although the exact mechanism is not known, several studies have shown an association between anabolic steroids and cardiovascular events $[6,7]$. In particular, atherogenic and thrombogenic effects of anabolic steroids as well as vasospastic and direct effects on myocardial damage have been described. Anabolic steroids may be themain reason or contributor of cardiovascular events. Chronic use of anabolic steroids may lead to hypertension, reduction in HDL cholesterol level and endothelial dysfunction, which are known to play part in the development of atherosclerosis. Increased thromboxane A2 receptor density, decreased production of prostaglandins, and increased levels of homocysteine and clotting factors have been found to be the leading mechanisms for arterial thrombosis, even in young healthy athletes. In addition to quantitative changes in the levels of anticoagulant and procoagulant factors, it has been shown that there is increased platelet sensitivity to collagens.

It is difficult to come to any definitive conclusions regarding the riskof venous thrombosis engendered by using a tourniquet [8]. The pneumatic tourniquet was introduced in 1904 by Harvey Cushing to maintain a bloodless filed during extremity surgery. Using a tourniquet is sometimes a necessity, and the possibility of deep venousthrombosis may become a relatively minor consideration when set against the need to provide the optimum conditions for essential surgery. But exsanguination and use of a tourniquet increases thechange of thrombus formation. Kroese and Stiris advises to avoid using a tourniquet in patients with particular risk, such as a history of venous thrombosis and patients with trauma to the lower limb $[9,10]$.

The most common complications in the clinical setting are neural complications, including ischemic neuropathy and compressive neurapraxia. Tourniquets may also cause direct vascular injury, particularly if the vessel wall is diseased. The tourniquet induces venous stasis and increased platelet adhesion in the valve pockets afterdistal limb ischemia.

Two hours or more of sustained ischemia during extremity surgery may lead to "post tourniquet syndrome", which is characterized by edema, stiffness, pallor, weakness without paralysis and numbness. The post tourniquet syndrome usually resolves within 1 week, although the recovery period may be prolonged.

Several prospective randomized clinical trials have shown no significant long-term deleterious effects of using them in extremity surgery.
Vascular complications are rare but potentially devastating events that are reported with varying frequencies during upper extremity regional anaesthesia [11]. Transient vascular insufficiency is a reported complication of brachial plexus blocks, occurring in up to $1 \%$ of patients. Vasospasm may follow arterial puncture or be a consequence of local anaesthetic-induced vasoconstriction. The risk of hematoma immediately after brachial plexus techniques is small $(0.001 \%-0.02 \%)$, although the incidence may increase at 1-month follow-up. Although most are inconsequential, hematomas have or have not been associated with postoperative paraesthesia or transient nerve injury. Pseudoaneurysm formation is another rare complication of brachial plexus block. Pressure- induced neural ischemia with subsequent neurological impairment may occur because of the close proximity of neurovascular structures withinthe axilla. Axillary artery dissection can result from intramural injection oflocal anaesthetic. In summary, vascular complications are rare after brachial plexus blockade but must be considered in patients with postoperative neurological impairment. Early recognition and prompt surgical intervention are critical to avoid longlasting neurological sequelae.

Complication after upper extremity deep venous thrombosis is the postthrombotic syndrome [2,3]. This is a chronic complication leading to significant functional disability and impaired quality of life, in particular when occurring in the dominant arm. Symptoms include edema, persistent pain or heaviness of the affected arm, limb fatigue with exertion, skin discoloration and distended collateral veins in the shoulder region. The 2 year cumulative incidence rates of recurrent venous thromboembolism after a first episode of upper extremity deep venous thrombosis range between $4 \%$ and $8 \%$. The actual incidence of pulmonary embolism resulting from upper extremity deep venous thrombosis is between $3 \%$ and $12 \%$.

Although it is generally accepted that, as for patients with lower extremity deep venous thrombosis, patients with (symptomatic) thrombosis of the arm veins require anticoagulant treatment [2, 3]. This prevents thrombus propagation and pulmonary embolism. But the optimal treatment duration and intensity is not determined in randomized controlled trials. The current guidelines recommend initial treatment with low molecular weight heparin (LMWH) or unfractionated heparin, followed by vitamin $\mathrm{K}$ antagonists.

In a case report by Singh et al., left proximal subclavian vein thrombosis was diagnosed in a 27-year-old male three days after a soft tissue injury in the left shoulder [12]. Urokinase was administered for the first 24 hours and treatment was continued with intravenous heparin and oral warfarin. Oral anticoagulation was continued for 12 months. The patient'ssymptoms resolved after the third month of medication.

Another case report by Basat et al, shows a 54-year-old male patient were pulmonary embolism with thrombus at the right brachial vein was diagnosed after a surgical treatment for ulnar pseudarthrosis. The patient was heparinized and on the second day LMW heparins were administered [13].

Peivandi presented a 25-year-old man with a simple displaced clavicle fracture at the middle third. (14) One week after trauma he was diagnosed with thrombus extending from the brachial axillary veins to the distal subclavian vein. Treatment with anticoagulation therapy was started.

In conclusion, it is important to be aware of this unusual but potentially serious complication, as early diagnosis and treatment may limit morbidity and mortality [15]. We must be aware of the symptoms of deep vein thrombosis of the upper extremity, and have a high index of suspicion.

\section{References}

1. SCHOLTEN RJPM., VAN DER MOLEN M., BERNARD MJ UITDEHAAG , LEX M BOUTER, HENRICA CW DE VET ,(2007), Surgical treatment options for carpal tunnel syndrome. Cochrane Database of Systematic Reviews: Issue 4.DOI: 10.1002/14651858.CD003905

2. SCHMELZER R., ROCCA G., GREGORY J. DELLA.; CAPLIN, DAVID A.; (2006), Endoscopic Carpal Tunnel Release: A Review of 753 Cases in 486 Patients. Plastic and Reconstructive Surgery: January. DOI: 0.1097/01.prs.0000194910.30455.16 
3. BENSON L., BARE A., (2006), Complications of Endoscopic and Open Carpal Tunnel Release. Arthroscopy: The Journal of Arthroscopic and Related Surgery, Vol 22, No 9 (September), pp 919-924. doi:10.1016/j.arthro.2006.05.008

4. CZIHAL M., HOFFMANN U. (2011), Upper extremity deep venous thrombosis. Vasc Med, 16: 191. DOI: $10.1177 / 1358863 X 10395657$

5. MAI C., HUNT D., (2011), Upper-extremity Deep Venous Thrombosis: A Review. The American Journal of Medicine: Vol 124, No 5, May. doi:10.1016/j.amjmed.2010.11.022

6. GLUECK C., GOLDENBERG.N, SHAAISTA BUDHANI., DANIELLOTNER., CESAR ABUCHAIBE, et al. (2011), Thrombotic events after starting exogenous testosterone in men with previously undiagnosed familial thrombophilia. Translational Research, October. doi:10.1016/j.trsl.2011.06.003

7. FERENCHICK G.S. (1991), Anabolic/Androgenic Steroid Abuse and Thrombosis: Is There a Connection? Medical hypotheses: 35, 27-31.

8. WAKAI A., WINTER D., Street, John T., Redmond, Paul H., Pneumatic Tourniquets in Extremity Surgery. Journal of the American Academy of Orthopaedic Surgeons, September/October 200, Vol 9, No 5.
9. KROESE Aj,STIRIS G,(1974), Does peroperativ-e pneumatic tourniquet cause thrombosis?Journal of Oslo City Hospital; 24:69-73.

10. KROESE Aj, STIRIS G., (1975), The risk of deep-vein thrombosis after operations on a bloodless lower limb. A xenographic study. Injury7: 271-3.

11. NEAL J., GERANCHER., JAMES R. HEBL.,BRIAN M. ILFELD., COLIN J.L. MCCARTNEY.,et al. (2009), Upper Extremity Regional Anesthesia. Reg Anesth Pain Med. Mar-Apr; 34(2): 134- 170.doi: 10.1097/AAP.0b013e31819624eb

12. SINGH A., SINGH A., Mahajan S, (2009), Upper extremity deep vein thrombosis following soft tissue trauma. Acta Orthop Traumatol Turc ; 43(4):376-378. doi:10.3944/AOTT.2009.376

13. BASAT H., KALEM M., MEHMET S. BINNET., MEHMET DEMIRTAS., (2011), Pulmonary thromboembolism after surgical treatment of ulnar pseudarthrosis: a case report. Acta Orthop Traumatol Turc; 45(4):284-287. doi:10.3944/AOTT.2011.2426

14. PEIVANDI M., NAZEMIAN Z., (2011), Clavicular Fracture and Upper-Extremity Deep Venous Thrombosis. Orthopedics, March Volume 34 - Issue 3: 227. DOI: 10.3928/01477447-20110124-28

15. KUCHER N. (2011), Deep-Vein Thrombosis of the Upper Extremities. $n$ engl j med, march 3, 364;9 